\title{
ESTRATÉGIAS DE ESTUDO E APRENDIZAGEM DE ALUNOS UNIVERSITÁRIOS: UM ESTUDO EXPLORATÓRIO
}

\section{ESTRATEGIAS DE ESTUDIO Y APRENDIZAJE DE ESTUDIANTES DE ENSEÑANZA SUPERIOR: UM ESTUDIO EXPLORATORIO}

\author{
Linete Bartalo - linete@uel.br \\ Doutora em Educação pela Universidade Estadual Paulista Júlio de Mesquita Filho \\ (UNESP/Marília). Professora do Departamento de Ciência da Informação da Universidade \\ Estadual de Londrina (UEL).
}

\section{Sueli Édi Rufini Guimarães - sueli rufini@hotmail.com}

Doutora em Educação pela Universidade Estadual de Campinas (UNICAMP). Professora do

Departamento de Educação da Universidade Estadual de Londrina (UEL)

\section{Resumo}

O desempenho acadêmico e a motivação de estudantes do ensino superior têm sido relacionados com $O$ uso adequado de estratégias de estudo e de aprendizagem. A literatura indica a possibilidade de se aprender a utilizar estratégias de estudo e aprendizagem, com resultados favoráveis para o envolvimento acadêmico. Neste estudo, de caráter exploratório, foram levantados dados acerca de um contexto ainda não investigado nesta temática. Seu mérito é apresentar um panorama do uso de estratégias de estudo e aprendizagem por alunos de cursos superiores. Investigou-se o uso de estratégias de estudo e aprendizagem de uma amostra de 109 estudantes dos cursos de Arquivologia e Biblioteconomia de duas instituições públicas dos estados de São Paulo e Paraná. Os dados foram coletados mediante versão do LASSI (Learning and Study Strategies Inventory) validada para o Brasil e os resultados indicaram que os estudantes valorizam seus cursos e os relacionam com metas futuras. Além disso, foi identificado um baixo desempenho na avaliação do gerenciamento do tempo. Foram descobertas diferenças significativas na comparação entre 0 desempenho dos alunos dos referidos cursos com o de estudantes de 17 outros cursos de graduação, em diversas categorias contidas no LASSI. Discutem-se as possibilidades de intervenção, sendo o ensino do uso de estratégias de estudo e aprendizagem uma alternativa pedagógica viável, com importantes implicações educacionais.

\section{Palavras chave}

Estratégias de Estudo, Estratégias de Aprendizagem, Ensino Superior, LASSI. 


\section{INTRODUÇÃO}

É generalizada a queixa de professores universitários a respeito da falta de interesse e dedicação aos estudos de seus alunos. Alguns se dizem interessados, mas, freqüentemente, justificam o pouco empenho pela falta de tempo para se envolverem suficientemente nas atividades de seus cursos. Assim, de modo geral, no ensino superior, os estudantes adotam atitudes passivas diante da aprendizagem, esperam que seus professores assumam um papel ativo, apresentando argumentos convincentes para que seus alunos estudem determinados conteúdos, organizando aulas interessantes e, quem sabe, divertidas.

Em parte, essas expectativas representam a continuidade de uma postura aprendida na vida escolar, geralmente baseada em um modelo de transmissão de informações, no qual o professor é a figura de autoridade que indica o que deve ser aprendido e quais estratégias devem ser utilizadas para alcançar os objetivos estabelecidos (COVINGTON, 2004).

Por outro lado, os professores universitários continuam privilegiando $\mathrm{O}$ clima de passividade mediante a preferência por aulas expositivas, atividades pré-determinadas e por modelos avaliativos que cobram meramente o que foi transmitido (BZUNECK, 2004; 2005). Mesmo assim, esperam que seus alunos sejam ativos, questionadores, autoregulados sem, no entanto, haver orientações acerca das habilidades ou das estratégias necessárias para que trabalhem de modo independente. De acordo com Tollefson (2000), é do professor a responsabilidade de superar os desencontros entre as expectativas trazidas ou geradas no ambiente escolar, apontando, entre outras alternativas, o conhecimento de estratégias diversificadas de ensino e de aprendizagem.

A aprendizagem significativa, postulada por Ausubel (1963, 1968), tem sido resgatada por educadores preocupados, entre outras questões, com a perspectiva de formação do pensamento crítico e criativo, com a responsabilidade do aprendiz em construir seu conhecimento, com a importância e o valor atribuídos ao aprender a aprender e com a urgência em formar e manter cidadãos responsáveis e independentes. Noguerol (1999) e Pontes Neto (2001), entre outros pesquisadores, chamam a atenção para a importância do domínio, por parte dos alunos, das estratégias de estudo e aprendizagem com vistas a uma aprendizagem mais significativa. Nessa perspectiva, a aprendizagem pressupõe o protagonismo dos sujeitos e o objeto do conhecimento configura-se num 
organizador do diálogo entre quem aprende e quem ensina. Nesse sentido, a docência implica no reconhecimento de que existem saberes docentes, distintos dos saberes dos alunos. Partindo da premissa de que o professor é "aquele que pensa com o outro", a prática pedagógica exige que a palavra dos alunos seja ouvida, criando-se um espaço em que possam trazer e explicitar seus conhecimentos prévios, uma vez que é a partir deles que se iniciam novas aprendizagens.

É possível auxiliar os estudantes a se tornarem ativos, exercendo controle e refletindo a respeito da própria aprendizagem, por meio do ensino de estratégias de estudo e aprendizagem. Tais estratégias são seqüências integradas de procedimentos ou de atividades que o indivíduo escolhe com o propósito de facilitar a aquisição, o armazenamento e/ou a utilização da informação ou do conhecimento. Podem ser vistas como um conjunto mental de operações interdependentes que são trocáveis entre si e que podem ser modificadas em resposta a diferentes situações. (BORUCHOVITCH, 2001; PORTILHO, 2004; STEDILE, 2004).

O uso adequado dessas estratégias pode contribuir para se evitar a perspectiva homogeneizante que geralmente norteia 0 trabalho de sala de aula, no qual o professor planeja e executa ações educativas para um grupo de alunos que, idealmente, apresenta os mesmos conhecimentos, ritmos de aprendizagem e interesses, entre outras características. A superação desta perspectiva, por meio da valorização das estratégias de estudo e aprendizagem, pode ampliar as possibilidades de diálogo no grupo, na medida em que a sua utilização promove o que se denomina processamento de profundidade da informação, em oposição às formas de processamento de superfície. (CRAIK; LOCKART, 1972; ENTWISTLE; WATERSON, 1988).

Apesar da importância do uso de estratégias eficazes de aprendizagem, um estudante, ao realizar uma atividade acadêmica, raramente define antecipadamente que estratégias serão utilizadas. Não se preocupa quanto ao tempo que será despendido, não prepara o ambiente de aprendizagem, não seleciona os materiais que necessitará, não tem clareza sobre os objetivos que deseja alcançar, enfim, não planeja a sua aprendizagem. Pensa no objetivo em si, ou seja, apropriar-se daquele conhecimento, realizar a tarefa, dialogar com o grupo, mas o como, o meio, as formas não são pensadas antecipadamente ou, quando o são, via de regra, acontecem de modo não sistemático.

Antonijevic e Chadwick (1982) entendem que, para a maioria das pessoas, a aprendizagem ocorre quase 
inconscientemente, porém, assinalam que os melhores aprendizes são justamente aqueles que têm um nível mais alto de consciência do processo. $\mathrm{O}$ uso de estratégias de estudo e aprendizagem é sempre consciente $\mathrm{e}$ intencional $\mathrm{e}$, por essa razão, são chamadas de estratégias cognitivas de aprendizagem, por se realizarem mediante processos mentais. As estratégias metacognitivas, referem-se ao conhecimento e à consciência desses processos mentais, o que permite seu monitoramento, sua avaliação e regulação.

Alguns autores (ANTONIJEVIC; CHADWICK, 1982; POZO MUNICIO apud PORTILHO, 2004; SIMÃO, 2001) fazem a diferenciação entre estratégias de aprendizagem e técnicas de estudo. Para eles, as técnicas são procedimentos que se aplicam de modo não controlado, não planejado e rotineiro, ao passo que as estratégias devem ser planejadas e controladas para serem executadas. Já Noguerol (1999) utiliza ambos os termos como sinônimos. Azevedo (1993) refere-se a "táticas", esclarecendo que é um termo de alcance menos geral que estratégias, podendo uma estratégia implicar o uso de táticas diversas.

Uma

desenvolvimento de uma área de conhecimento é a elaboração de instrumentos para coleta de dados. Em relação às estratégias de estudo e aprendizagem, Figueira (1994) traduziu e adaptou para o português o LASSI (Learning and Study Strategies Inventory) e o validou para uma amostra de estudantes portugueses. Bartalo (2006) levantou as propriedades psicométricas da versão brasileira do mesmo instrumento, confirmando a inclusão de oito categorias de estratégias de aprendizagem, são elas:

1) Processamento da Informação: avalia a utilização de elaboração imagética e verbal, monitoração da compreensão e do raciocínio e, de modo geral, a criação de pontes entre o que já sabem e o que estão tentando aprender e se lembrar, i.e., aquisição de conhecimento, retenção e aplicação futura (p. ex. "Tento encontrar ligações entre o que estou estudando e as minhas próprias experiências"). Estudantes com baixos escores nessa categoria necessitam aprender métodos que o auxiliem a tornar a aprendizagem mais significativa e organizada. Tais métodos podem ir desde o simples parafrasear e sumarizar, até à criação de analogias, de esquemas e de notas, bem como a utilização de raciocínio inferencial, analítico e sintético.

2) Ansiedade: mede o grau de preocupação com a escola e com seu desempenho acadêmico (p. ex. "Fico tão nervoso e confuso quando faço uma prova que as respostas que dou 
não são as melhores que a minha capacidade permite"). Estudantes com baixos escores nessa categoria estão experimentando níveis altos de ansiedade, associados com a escola e com seu desempenho e podem precisar desenvolver técnicas para controle da ansiedade e redução da preocupação, de forma que a atenção possa ser focalizada para as tarefas.

3) Organização do tempo: avalia a aplicação de princípios de administração de tempo a situações acadêmicas (p. ex. "Acho difícil cumprir um horário de estudo"). Estudantes com baixos escores nessa categoria, podem precisar desenvolver programação efetiva e técnicas de monitoração do tempo para assegurar a conclusão de tarefas acadêmicas e evitar procrastinação, inclusive para atividades não acadêmicas.

4) Concentração: avalia a habilidade para dirigir e manter atenção em tarefas acadêmicas (p. ex. "A minha imaginação divaga muito quando estou fazendo trabalhos escolares"). Baixos escores nessa categoria podem indicar necessidade de aprender a monitorar o nível de concentração e desenvolver técnicas para redirecionar a atenção e eliminar pensamentos ou sentimentos que estejam interferindo de forma a dificultar a concentração.

5)Atitude: refere-se aos valores que estudantes atribuem à escola, bem como 0 interesse que têm pelo sucesso acadêmico, com vistas a que esse sucesso possa ajudá-los a arrumar um bom emprego (p. ex. "Não me importa concluir este curso, desde que consiga arranjar um bom emprego"). Estudantes com baixos escores nessa categoria podem não acreditar que a faculdade ou o curso que estão fazendo seja pertinente ou importante a eles e podem precisar desenvolver um entendimento melhor de como a escola e o seu desempenho acadêmico relacionamse com as suas metas de vida futura.

6)Seleção de Idéias Principais: avalia a habilidade de identificar informação importante entre informações menos importantes e detalhes, tanto em sala de aula como em estudos autônomos, para posterior utilização (p. ex. "Acho difícil saber o que é importante recordar de um texto de estudo"). Estudantes com baixa pontuação nessa categoria podem precisar desenvolver a habilidade de separar informação relevante na qual focalizar a atenção e as estratégias de processamento da informação. 
7) Auxiliares de Estudo: examina o grau de utilização, bem como de criação de técnicas de suporte ou materiais que possam auxiliar 0 estudante a aprender e a recordar a nova informação (p. ex. "Uso meios auxiliares para estudar como grifar as partes mais importantes do texto, escrever palavras chave ao lado dos parágrafos, fazer resumos etc."). Alunos que obtêm baixos escores nessa categoria necessitam aprender mais sobre auxiliares de estudo e qual a sua utilidade prática $e$, também, a criar seus próprios métodos de estudo e aprendizagem.

8) Motivação: avalia a diligência de estudantes, a autodisciplina e a força de vontade para trabalhar com afinco e a disponibilidade para assumir exigências acadêmicas completas ( $p$. ex. "Quando a matéria é difícil desisto de estudar ou só estudo as partes fáceis"). Estudantes com baixos escores nessa categoria devem aprender como fixar e usar metas para tornarem-se capazes de realizar tarefas específicas.

Neste artigo são relatados os resultados de uma pesquisa que identificou o uso de estratégias de estudo e aprendizagem de alunos de cursos de Arquivologia e Biblioteconomia de duas universidades públicas. Além disso, buscou-se comparar o desempenho desses estudantes com o obtido por outros, oriundos de diversos cursos de graduação de quatro universidades públicas. A justificativa desta comparação baseia-se na possibilidade de se conhecer o perfil dos estudantes dessa área de conhecimentos acerca do uso de estratégias de estudo e aprendizagem. Os resultados são analisados em termos das implicações educacionais, podendo propiciar conhecimentos que representem alternativas para possíveis intervenções pedagógicas.

\section{METODOLOGIA}

\subsection{Participantes}

Participaram 109 alunos das terceiras séries dos cursos de Biblioteconomia e Arquivologia de duas universidades públicas, localizadas em cidades do interior dos estados do Paraná e de São Paulo. As idades dos participantes variaram de 19 a 45 anos, com uma média de 27 anos aproximadamente; sendo 83 do gênero feminino e 26 do masculino.

\subsection{Instrumento}

Utilizou-se como instrumento de coleta de dados um questionário, originariamente desenvolvido na Universidade do Texas (WEINSTEIN; ZIMMERMAN; PALMER, 1988), traduzido 
e adaptado por Figueira (1994) e de uso e com assinatura de termo de corrente, o LASSI (Learning and Study consentimento livre e esclarecido pelos Strategies Inventory), validado no Brasil alunos participantes.

por Bartalo (2006). Constitui-se em um

inventário composto por 88 questões em escala likert de 5 pontos, desde 1 "nada característico" até 5 "totalmente característico" e contempla 8 categorias de estratégias de estudo e aprendizagem: Processamento da Informação, Ansiedade, Organização do Tempo, Concentração, Atitude, Seleção de Idéias Principais, Auxiliares de Estudo e Motivação.

Das 88 questões contidas no instrumento, 11 foram elaboradas para avaliar o uso de duas destas categorias (Processamento da Informação e Seleção de Idéias Principais) quando o participante utiliza a Internet.

Os dados foram coletados em situações de sala de aula, com a autorização das coordenações dos cursos

\subsection{Procedimentos}

Os dados foram tabulados e processados utilizando-se o programa Statistica 7.0. Para alcançar os objetivos propostos, foram realizadas estatísticas descritivas e análise de variância.

\section{RESULTADOS}

A Tabela 1 apresenta os resultados de desempenho dos participantes nas avaliações de cada categoria contida no instrumento. Observa-se que o maior desempenho em ambos os cursos foi na avaliação da categoria Atitude e o menor na categoria Organização do Tempo.

Tabela 1 - Desempenho dos alunos dos cursos de Arquivolovogia e Biblioteconomia na avaliação das estratégias de estudo e aprendizagem

\begin{tabular}{lcccccc}
\hline & Cursos & \multicolumn{3}{c}{ Arquivologia } & \multicolumn{3}{c}{ Biblioteconomia } \\
Estratégias & N & M & Dp & N & \multicolumn{1}{c}{ M } & Dp \\
\hline Processamento da Informação & 47 & 3,59 & 0,73 & 57 & 3,64 & 0,65 \\
Ansiedade & 49 & 3,36 & 0,90 & 59 & 3,32 & 0,81 \\
Organização do Tempo & 49 & 2,82 & 0,85 & 59 & 2,84 & 0,67 \\
Concentração & 49 & 3,51 & 0,89 & 59 & 3,62 & 0,82 \\
Atitude & 49 & 4,25 & 0,78 & 59 & 4,26 & 0,66 \\
Seleção de Idéias Principais & 48 & 3,65 & 0,91 & 58 & 3,50 & 0,77 \\
Auxiliares de Estudo & 50 & 2,94 & 0,90 & 59 & 3,16 & 0,80 \\
Motivação & 50 & 3,59 & 0,72 & 59 & 3,53 & 0,68 \\
\hline N número de participantes & & & & & & \\
M Média & & & & & &
\end{tabular}

Buscando atender ao objetivo de procuradas diferenças no desempenho dos conhecer o perfil dos estudantes de participantes na avaliação do uso de Arquivologia e de Biblioteconomia foram estratégias pela análise de variância. 
Inicialmente,

comparando-se

desempenho nas categorias avaliadas com

a idade dos participantes, não foram encontradas diferenças estatisticamente significativas. $\mathrm{Na}$ comparação com $\mathrm{O}$ gênero, o desempenho das mulheres foi superior ao dos homens sendo $F(1,59)=4,62$, com $p \leq a 0,03$. Também foi significativamente superior o desempenho das mulheres na avaliação da categoria Motivação, com $F(1,59)=6,09 p \leq a$ 0,01.

$\mathrm{Na}$ comparação entre o desempenho na avaliação das categorias e a instituição de ensino dos participantes, não foram encontradas diferenças estatisticamente significativas.

Em outra linha de análise, por meio de Análise de Variância, o desempenho dos participantes dos dois cursos foi comparado ao de alunos de 17 outros cursos de graduação de quatro instituições públicas de ensino superior, sendo duas do interior do estado do Paraná e duas do interior do estado de São Paulo. Os outros 17 cursos são: Enfermagem, Ciência da Computação, Engenharia Civil, Pedagogia, Odontologia, História, Letras, Ciências Contábeis, Administração, Biotecnologia, Ciências Biológicas, Filosofia, Ciências Sociais, Fisioterapia, Terapia Ocupacional, Fonoaudiologia e Relações Internacionais.

Os resultados do teste pos hoc de Tukey indicaram que os estudantes do curso de Arquivologia tiveram desempenhos significativamente inferiores na avaliação do uso de estratégias de Processamento da Informação, comparados com alunos dos cursos de Pedagogia $(p=0,03)$, Odontologia $(0,04)$ e Fisioterapia (0,02). Também foram inferiores os resultados de seu desempenho na avaliação do uso de estratégias de Concentração, comparados aos estudantes de Pedagogia $(0,01)$, Fisioterapia $(0,02)$ e Fonoaudiologia $(0,02)$. Finalmente, foi significativamente inferior $o$ desempenho dos alunos de Arquivologia na avaliação do uso de estratégias de Motivação, comparados com o desempenho dos alunos dos cursos de Pedagogia $(0,02)$, Odontologia $(0,001)$, Fisioterapia $(0,0001)$ e Fonoaudiologia $(0,002)$.

Seguindo a mesma análise, o desempenho dos estudantes do curso de Biblioteconomia foi significativamente superior ao obtido pelos estudantes dos cursos de Ciência da Computação $(0,02)$ e Engenharia Civil $(0,04)$, no uso das estratégias de Organização do Tempo. Foram significativamente inferiores as médias obtidas pelos estudantes de Biblioteconomia na avaliação do uso de estratégias de Concentração, quando comparadas com as médias dos estudantes de Pedagogia $(0,01)$ e Fisioterapia $(0,02)$. 


\section{POSSÍVEIS CONSIDERAÇÕES}

Traçar o perfil dos alunos a respeito do uso de estratégias de estudo e aprendizagem dos dois cursos investigados, por se tratar de uma mesma área de conhecimentos, foi um dos propósitos desta investigação. $\mathrm{O}$ que se evidencia nos dados coletados é que os estudantes valorizam seu curso, seu desempenho acadêmico e acreditam na possibilidade de obterem com ele oportunidade de trabalho futuro. Este resultado está alinhado com o encontrado por Alcará (2007) que analisou a motivação e o uso de estratégias de aprendizagem e perspectiva de tempo futuro de estudantes de Biblioteconomia. Esta última variável, perspectiva de tempo futuro, refere-se à adoção de uma meta, colocada num futuro próximo, que pode relacionar-se de modo positivo com o envolvimento, no presente, em atividades de aprendizagem. No caso dos estudantes de biblioteconomia, o estudo revelou que os mesmos têm metas futuras de trabalho as quais foram relacionadas com a qualidade autônoma de sua motivação para os estudos e com o uso de estratégias de aprendizagem. Além disso, o fato de fixarem metas valorizadas para um futuro próximo esteve relacionado com a percepção de instrumentalidade das atividades presentes, ou seja, as tarefas acadêmicas, de modo geral, foram percebidas como úteis para o alcance das metas colocadas no futuro. Comparandose com os resultados do presente estudo, incluindo aqui mais um curso de graduação da mesma área de conhecimento, parece que os alunos têm atitudes positivas em relação ao seu curso pelo fato de o consideraram um meio importante para alcançarem boas oportunidades de trabalho após sua formatura. Em termos motivacionais, quando uma meta futura é fixada de modo autônomo, isto é, por vontade própria e com liberdade psicológica, sem coação externa, ela pode influenciar de modo favorável o envolvimento dos estudantes no presente. Aqui, o envolvimento refere-se ao fato de selecionar estratégias para trabalhar de modo eficaz nas atividades acadêmicas.

A avaliação da estratégia de gerenciamento do tempo foi a categoria na qual os participantes dos dois cursos obtiveram o desempenho mais baixo. Partindo-se da premissa de que a busca de uma administração do tempo mais eficiente é preocupação da maioria das pessoas, sendo ou não alunos universitários, este dado precisa ser analisado com atenção. Administrar o tempo disponível, principalmente se considerar que parte dos universitários divide-se entre atividades acadêmicas e de trabalho, é fundamental para um bom desempenho. Os baixos resultados encontrados permitem inferir que os alunos investigados podem estar com desempenhos aquém de suas reais 
possibilidades, considerando-se maior disponibilidade de tempo, e um gerenciamento mais adequado. Parece um tanto incoerente, vinculando este ao resultado dos participantes na avaliação obtida na categoria Atitude, pois, caso valorizem seus estudos, deveriam concentrar-se na organização de condições adequadas para uma aprendizagem eficaz. Isto pode significar que eles não aprenderam como se organizar, deixando um espaço importante para intervenção pedagógica. Como afirmam Boruchovitch e Bzuneck (2004), as estratégias de estudo e aprendizagem podem e devem ser conteúdo de ensino, tendo em vista a possibilidade de mudança nos alunos, quando aprendem novas alternativas de trabalho. Cursos existem que se propõem a ensinar as pessoas a administrar o seu tempo. Pesquisas realizadas com universitários têm entre seus resultados, descobertas a respeito deste construto (BARTALO, 1997; 2006; DICKISON; O'CONNEL, 1990).

O fato dos participantes desta pesquisa serem oriundos de uma mesma área de conhecimento, sugeriu, a princípio, por suposição, um padrão coerente na avaliação do uso de estratégias de estudo e aprendizagem, pois aquele fato poderia resultar numa organização similar do ambiente de aprendizagem, o que não se confirmou nos resultados obtidos. Ames (1992) assinalou a importância das estruturas de ensino nas salas de aula, uma vez que elas são provenientes da cultura e dos valores compartilhados pelos seus atores, alunos e professores. A comparação entre o desempenho dos estudantes de Arquivologia e Biblioteconomia com o de estudantes de 17 outros cursos de graduação revelou que não se pode considerar, nesta amostra, que os dois cursos têm um perfil semelhante.

Os estudantes de Arquivologia apresentaram resultados mais baixos em diversas categorias de estratégias de estudo e aprendizagem, como Processamento da Informação, Concentração e Motivação, comparados com o desempenho de estudantes das áreas de Ciências da Saúde e de Ciências Humanas.

No caso dos estudantes de Biblioteconomia, os resultados foram mais próximos do total de alunos, ou seja, foram identificadas poucas diferenças entre seus desempenhos e 0 de estudantes dos demais cursos. Além disso, na categoria Organização do Tempo, o desempenho foi superior ao de dois cursos da área de Ciências Exatas. Apenas na avaliação da categoria Concentração seu desempenho foi inferior a dois outros cursos.

Apesar de não se revelarem como um grupo homogêneo em relação ao uso de estratégias de estudo e aprendizagem, algumas considerações podem ser feitas 
no sentido de estimular o seu uso.

Boruchovitch (1999) ressalta a importância que o ensino de estratégias de aprendizagem tem para a melhoria do desempenho escolar, enfatizando a eficácia da manipulação de tais estratégias para diminuir as dificuldades pessoais dos alunos, maximizar a aprendizagem e controlar melhor os fatores ambientais que interferem no desempenho escolar satisfatório.

Acredita-se que todo aluno, ao entrar para a universidade, possua um repertório anterior de estratégias de aprendizagem, as quais foram se sedimentando ao longo de sua vida acadêmica anterior. Esse repertório, aprendido em diversas e diferentes situações, foi adquirido graças a experiências vivenciadas, tanto em contextos informais quanto formais de educação. De acordo com os resultados desta pesquisa, os estudantes podem se desenvolver, caso tenham a oportunidade de conhecer e aprender a utilizar as estratégias de estudo e aprendizagem.

Estudos de natureza exploratória e correlacional, como o aqui apresentado, têm o mérito de trazer informações acerca de situações ainda não conhecidas, no caso, as estratégias utilizadas por alunos de uma área de conhecimento. Os resultados aqui descritos podem oportunizar o delineamento de outras investigações e, ainda, o planejamento de atividades de intervenção pedagógica.

\section{REFERÊNCIAS}

ALCARÁ, A. R. Orientações motivacionais de alunos do curso de biblioteconomia de uma universidade pública do norte do Paraná. 2007. 122 f. Dissertação (Mestrado em Educação) - Universidade Estadual de Londrina, Londrina, 2007.

AMES, C. Classroom: goals, structures, and student motivation. Journal of Educational Psychology, Arlington, v. 84, n. 3, p. 261-271, 1992.

ANTONIJEVIC, N.; CHADWICK, C. Estrategias cognitivas y metacognición. Revista de Tecnología Educativa, Santiago (Chile), v. 7, n. 4: p. 307-321, 1981/82.

AUSUBEL, D. P. The psychology of meaningful verbal learning. New York: Grune \& Stratton, 1963.

AUSUBEL, D. P. Educational psychology: a cognitive view. New York: Holt, Rinehart and Winston, 1968.

AZEVEDO, M. Estratégias de estudo e aprendizagem. Universidade de Lisboa, Faculdade de Ciências, Departamento de Educação. 1993. Disponível em: $<$ http://www.educ.fc.ul.pt/docentes/mazeve do/materiais/M\%C3\%A9todosDeEstudo_08 -09/Aprendiz06Estrateg.pdf>. Acesso em: 21 abr. 2004.

BARTALO, L. Leitura, hábitos de estudo e desempenho acadêmico de estudantes de Biblioteconomia (UEL). 1997, $189 \mathrm{f}$. Dissertação (Mestrado em Educação) Universidade Estadual de Londrina, Londrina, 1997. . Mensuração de estratégias de estudo e aprendizagem de alunos universitários : learning and study strategies inventory (LASSI) adaptação e validação para o Brasil. 2006, 215 f. Tese (Doutorado em Educação) - Universidade Estadual Paulista Julio de Mesquita Filho, Marília, 2006. 
BORUCHOVITCH, E. Estratégias de aprendizagem e desempenho escolar : considerações para a prática educacional. Psicologia: Reflexão e Critica, Porto Alegre, v. 12, n.2, 1999. Disponível em $<$ http://www.scielo.br/scielo.php?script=sci arttext\&pid=S0102-

$79721999000200008 \& \operatorname{lng}=e n \& n r m=i s o>$. Acesso em: 29 mar. 2005.

Dificuldades de aprendizagem, problemas motivacionais e estratégias de aprendizagem. In: SISTO, F. F. et al. (Org.) Dificuldades de aprendizagem no contexto psicopedagógico. 2. ed. Petrópolis: Vozes, 2001. 235p. p. 40-59.

BORUCHOVITCH, E.; BZUNECK, J.A. (Org.) Aprendizagem: processos psicológicos e o contexto social na escola. Petrópolis: Vozes, 2004.

BZUNECK, J. A. Aprendizagem por processamento da informação: uma visão construtivista. In: BORUCHOVITCH, E.; BZUNECK, J. A. (Org.). Aprendizagem: processos psicológicos e o contexto social na escola. Petrópolis: Vozes, 2004. p. 1754.

A motivação dos alunos em cursos superiores. In: JOLY, M. C. R. A.; SANTOS, A. A. A.; SISTO, F. F. (Org.). Questões do cotidiano universitário. São Paulo: Casa do Psicólogo, 2005, p. 217237.

COVINGTON, M. V. Self-worth theory: goes to college or do our motivation theories motivate? In: MCINERNEY, D. M.; VAN ETTEN, S. (Ed.) Big theories revisited. Greenwich, Conn: Age

Publishing, 2004. p. 91-114,

CRAIK, F. I. M.; LOCKART, R. S. Levels of processing : a framework for memory research. Journal of Verbal Learning and Verbal Behavior, New York, v. 11, p. 671684, 1972.

DICKINSON, D. J.; O'CONNEL, D. Q. Effect of quality and quantity of study on students grades. Journal of Educational
Research, Washington, v. 93, n. 4, p. 227231, Mar./Abr. 1990.

ENTWISTLE, N.; WATERSON, S. Approaches to studying and levels of processing in University students. British Journal of Educational Psychology, Leicester, v. 58, p. 258-265, 1988.

FIGUEIRA, A. P. C. Inventário de estratégias de estudo e de aprendizagem Learning and Study Strategies Inventory LASSI (Weinstein e Palmer, 1987) Estudos de validação e adaptação. Psychologica, Coimbra, n. 12, p. 79-114, 1994.

NOGUEROL, A. Aprender na escola: técnicas de estudo e aprendizagem. Tradução Jussara Haubert Rodrigues. Porto Alegre: Artes Médicas, 1999. 168 p.

PONTES NETO, J. A. S. Sobre a aprendizagem significativa na escola. In: MARTINS, E. J. S. et. al. Diferentes faces da educação. São Paulo: Arte \& Ciência Villipress, 2001. p. 13-37.

PORTILHO, E. M. L. Como os alunos universitários gostam de aprender. Disponível em:

$<$ http://www.aprendervirtual.com/ver_notici a. php? codigo $=107>$. Acesso em: 20 abr. 2004.

STEDILE, N. L. R. Estratégias metacognitivas utilizadas para prevenir problemas de saúde. In: SEMINÁRIO DE PESQUISA EM EDUCAÇÃO DA REGIÃO SUL (ANPEDSUL), 5., 2004. Anais... Curitiba: ANPED, 2004. CD-ROM.

TOLLEFSON, N. Classroom applications of cognitive theories of motivation.

Educational Psychology Review, New York, v. 12, n. 1, p. 63-83, 2000.

SIMÃO, A. M. V. Integrar os princípios da aprendizagem estratégica no processo formativo dos professores. In: SEMINÁRIO DE MODELOS E PRÁTICAS DE FORMAÇÃO INICIAL DE PROFESSORES, 2001, Lisboa, PT. 
Anais... Lisboa: Universidade de Lisboa, Faculdade de Psicologia e de Ciências da Educação. Disponível em:

http://www.educ.fc.ul.pt/recentes/mpfip/pdfs lamvsimao.pdf>. Acesso em: 19 mar. 2004.

WEINSTEIN, C. E.; ZIMMMERMANN, S. A.; PALMER, D. R. Assessing learning strategies: the design and development of the Lassi. In: WEINSTEIN, C. E.; GOETZ, E. T.; ALEXANDER, P. A. (Ed.). Learning and study strategies: issues in assessment, instruction, and evaluation. New York:

Academic Press, 1988. p. 25-40.

\section{Title}

Learning and study strategies of university students: an exploratory study.

\section{Abstract}

Higher education students' academic performance and motivation have been related to the adequate use of learning and study strategies. Literature shows the possibility of study and learning strategies with favorable results for the academic involvement. In this exploratory research, data were collected on a new context. The innovative merits of the research are related to the presentation of an overview of the strategies which are used during studying and learning process by the students of high degree courses. A sample of 109 students of the Archive Sciences and Librarianship courses of two public institutions of the States of São Paulo and Paraná was investigated. Data were gathered based on the Brazilian version of LASSI (LEARNING AND STUDY STRATEGIES INVENTORY) and the results pointed out that the students value their courses and relate them with future goals. Furthermore, it was identified a low performance concerning time management. Significant differences were found in the comparison between the performance of students of the two courses and those of 17 other undergraduate courses, in different LASSI categories. Intervention possibilities are discussed, being the teaching of study and learning strategies use a viable pedagogical alternative, with important educational implications.

\section{Keywords}

Study Strategies, Learning strategies, Higher Education, LASSI.

\section{Título}

Estrategias de estudio y aprendizaje de estudiantes de enseñanza superior: um estudio exploratório.

\section{Resumen}

El rendimiento académico y la motivación de los estudiantes de enseñanza superior han sido relacionados con el uso adecuado de estrategias de estudio y aprendizaje. La literatura indica la posibilidad de la aprendizaje de estrategias con resultados favorables para la participación académica. En este estudio, de carácter exploratorio, los datos fueron recogidos en un contexto en que aún no se investigó este tema. Su mérito es presentar un panorama general de la utilización de estrategias para el estudio y el aprendizaje de los estudiantes de la enseñanza superior. Se investigó el uso de estrategias de estudio y aprendizaje de una muestra de 109 estudiantes de los cursos de Biblioteconomía y Arquivologia de dos instituciones públicas de los estados de Sao Paulo y Paraná. Los datos fueron recogidos por la versión de LASSI (Learning and Study Strategies Inventory) validada para el Brasil y los resultados mostraron que los estudiantes valoran sus cursos y los relacionam con metas futuras. También fue identificado un pobre desempeño en la gestión del tiempo. Se descubrieron diferencias significativas en la comparación entre el rendimiento de los alumnos de estos cursos, con alumnos de otros 17 cursos de graduación en varias categorías del LASSI. Se discute las posibilidades de intervención, considerando la enseñanza de la utilización de estrategias de estudio y aprendizaje, una alternativa pedagógica viable, con importantes repercusiones educativas.

\section{Palabras Clave}

Estrategias de Estudio, Estrategias de Aprendizaje, Enseñanza Superior, LASSI. 
Recebido em: 16.07.2008

Aceito em: 01.03.2009 